\title{
Nematóides em plantas ornamentais e floríferas em Lavras (MG)
}

\author{
MAURO J.N. COSTA ${ }^{1}$, SILVÉRIO J. COELHO ${ }^{2}$ e VICENTE P. CAMPOS ${ }^{1}$
}

\section{RESUMO}

Coletaram-se amostras de solo e/ou raízes de 56 plantas ornamentais e floríferas do Câmpus da Universidade Federal de Lavras (UFLA) (MG), com o objetivo de conhecer a ocorrência e a distribuição de nematóides nessas plantas. No laboratório, extraíramse nematóides livres no solo pela técnica de Jenkins. Pela trituração em liquidificador, obtiveram-se os nematóides sedentários. Xiphinema foi obtido através do peneiramento do solo e coletado entre detritos mediante a separação individual no microscópio de objetiva invertida. As freqüências das espécies de fitonematóides nas amostras foram: Helicotylenchus dihystera (19,7\%), Meloidogyne incognita (16,7\%), Xiphinema brasiliensi (10,61\%), Meloidogyne spp. (9,09\%), Ogma sp. (7,58\%), Trichodorus sp. (7,58\%), Meloidogyne javanica (7,58\%), Criconemella ornata (4,55\%), Tylenchorrynchus sp. (4,55\%), Aphelenchus sp. (3,03\%), Xiphinema spp. (3,03\%), Aphelenchus avenae (1,52\%), Tylenchulus semipenetrans $(1,52 \%)$, Dorilaimus sp. (1,52\%) e Ditylenchus sp. $(1,52 \%)$.

Palavras-chave: fitonematóides, plantas ornamentais e floríferas, levantamento.

\section{ABSTRACT \\ Nematodes in ornamental and flowering plant roots at Lavras, State of Minas Gerais, Brazil}

Soil and roots samples from ornamental and flowery plants were collected in the nursery of Agricultural Department of Federal Unversity of Lavras (UFLA). In the laboratory, free soil nematodes were extracted by Jenkins technique. By liquefier trituration, were obtained the sedentaries nematodes. The Xiphinema genus were obtained through soil sifting in the sieves mesh and it was collected among the dregs, through of individual separation at the microscope. The frequency of plant nematodes in the samples were: Helicotylenchus dihystera (19.7\%), Meloidogyne incognita (16.7\%), Xiphinema brasiliensi (10.61\%), Meloidogyne spp. (9.09\%), Ogma sp. (7.58\%), Trichodorus sp. (7.58\%), Meloidogyne javanica (7.58\%), Criconemella ornata (4.55\%), Tylenchorrynchus sp. (4.55\%), Aphelenchus sp. (3.03\%), Xiphinema spp. (3.03\%), Aphelenchus avenae (1.52\%), Tylenchulus semipenetrans (1.52\%), Dorilaimus sp. (1.52\%) and Ditylenchus sp. (1.52\%).

Key words: plant nematodes, flowery plants, ornamental plants, frequency.

\section{INTRODUÇÃO}

A produção de plantas é afetada por diferentes fatores, destacando-se, em muitas delas, os fitonematóides, que, além de causarem prejuízos às plantas pelo parasitismo, podem também tornar mais severas as enfermidades provocadas por outros microrganismos, principalmente fungos e bactérias (CAMPOS, 1992). Esses patógenos causam, na produção, perdas que variam de suaves até a destruição total. $\mathrm{O}$ grau de danos depende da suscetibilidade da cultura, das condições ambientais, da presença de outros patógenos que podem interagir com os nematóides e da densidade populacional dos nematóides encontrados (TIHOHOD, 1973). Essa densidade pode variar durante todo o ano nos climas tropicais, em face das variações de temperatura e da umidade do solo, poden-

\footnotetext{
${ }^{1}$ Universidade Federal de Lavras, Departamento de Fitopatologia. Caixa Postal 37, 37200-000 Lavras (MG). E-mail: mjncosta@zipmail.com.br

${ }^{2}$ Departamento de Fitotecnia.
} 
do até, no período frio ou de seca, ocorrer a dormência dos nematóides (LORDELLO, 1964).

Segundo CHASE et al. (1983), os nematóides que infestam raízes de plantas ornamentais ou floríferas incluem Meloidogyne spp. (nematóide das galhas), Pratylenchus spp. (nematóide das lesões), Radopholus spp. (nematóide cavernícola), Rotylenchulus reniformis (nematóide reniforme) e Cactodera spp. (nematóide dos cistos e de Cactus). Em adição a estes, Aphelenchoides spp., nematóide foliar, que comumente infesta a parte aérea de algumas plantas ornamentais. Os nematóides das galhas, Meloidogyne arenaria, $M$. incognita e $M$. javanica, causam danos em várias plantas ornamentais de acordo com LEHRMAN (1979), como Tradescantia fuscata, Brassaia actinophylla, Caladium bicolor, Maranta leuconeura, Philodendron spp., Sansevieria trifasciata e Siderasis fuscata. Plantas ornamentais atacadas pelo nematóide das lesões Pratylenchus coffeae e $P$. penetrans sofrem danos significativos como a Aglaonema commutatum, Caladium spp, Chamaedorea seifrizii, Codiaeum variegatum, Dracaena spp., Monstera deliciosa, Philodendron bipinnifolium e Singonium podophyllum. CORBETTI (1983) cita a disseminação do nematóide das lesões (Pratylenchus bolivianus) na Holanda, mediante rizomas da planta ornamental alstroeméria (Alstroemeria spp., fam. Amaryllidaceae), levados da América do Sul. O nematóide cavernícola, Radopholus similis, é um sério patógeno em muitos países tropicais e subtropicais. Nos EUA, é problema em casas de vegetação de plantas ornamentais nos seguintes Estados: Arizona, Califórnia, Havaí, Luisiânia e Texas (MARTIN, 1978). O nematóide reniforme (Rotylenchulus reniformis) tem impedido, de forma significativa, o desenvolvimento da indústria ornamental, porque plantas contaminadas ou infestadas por ele são sujeitas a quarentena no Arizona, no Novo México e na Califórnia, o que resulta em impacto no comércio de flores (INSERRA et al., 1981). O uso de palmeiras ornamentais tem aumentado significativamente no Sul da Flórida, cujas condições climáticas são extremamente adequadas ao seu desenvolvimento. Muitas delas, no entanto, são disseminadoras do nematóide reniforme, por meio do transplantio sistemático dessas plantas, algumas delas infestadas pelo patógeno. $\mathrm{O}$ nematóide dos cistos, Cactodera cacti (Syn. Heterodera cacti) aparentemente não causa danos significativos em Schlumbergena truncata e muitas espécies de Cactus, nas muitas áreas analisadas (SOUTHEY, 1957).
Apesar dos grandes avanços nas técnicas de extração e quantificação de fitonematóides, possibilitando a investigação da magnitude da sua população no hospedeiro, ainda não se pesquisaram, no Brasil, de forma quantitativa, os efeitos da interação nematóide-plantas ornamentais e nematóide-plantas floríferas. O conhecimento da população de fitonematóides é de grande importância, pois serve de base para futuros estudos sobre a diagnose, densidades populacionais e aplicação de medidas de controle. Dessa forma, objetivou-se, neste trabalho, fazer levantamento da incidência de fitonematóides em espécies ornamentais e floríferas.

\section{MATERIAL E MÉTODOS}

Canteiros com plantas ornamentais ou floríferas e solo ao redor de plantas arbustivas do setor de Paisagismo e Floricultura-DAG/UFLA, foram amostrados em diversos pontos. Tanto nos canteiros como ao redor de plantas arbustivas, procedeu-se à capina para retirada de plantas daninhas. O procedimento para a amostragem incluiu a utilização de trado de 5 $\mathrm{cm}$ de diâmetro, introduzido a $15-20 \mathrm{~cm}$ de profundidade na projeção da copa da plantas, retirando-se porção de solo que foi colocado em saco plástico, constituindo uma subamostra. Colocaram-se três subamostras assim coletadas por planta, em saco plástico devidamente identificado, constituindo uma amostra composta. Em cada análise laboratorial, tal amostra foi dividida em três subamostras de 100 gramas cada uma, as quais foram processadas no Laboratório de Nematologia do Departamento de Fitopatologia/UFLA.

A extração dos nematóides foi feita logo em seguida à coleta. No caso do solo, utilizou-se a técnica do peneiramento, seguido de centrifugação em solução de sacarose (JENKINS, 1964), ou de peneiramento combinado com o método de Baerman modificado (FLEGG \& HOOPER, 1970). Os nematóides das raízes foram extraídos por método proposto por COOLEN \& D'HERDE (1972), incluindo mecanicamente a desintegração de raízes, na presença de água, seguida de peneiramento e centrifugação em solução de sacarose.

Com os nematóides em água, procedeu-se à identificação mediante montagem dos espécimes em lâminas semipermanentes e uso de chaves de classificação atualizadas. Efetuou-se a identificação das espécies de Meloidogyne com base no exame da configuração perineal das fêmeas, segundo a técnica de HARTMAN \& SASSER (1985). 


\section{RESULTADOS E DISCUSSÃO}

Das amostras de 56 plantas analisadas, 27 apresentaram nematóides. Diferentes gêneros foram en- contrados associados às plantas, predominando Meloidogyne e Helicotylenchus.

Algumas espécies de plantas não apresentaram infestação, enquanto outras apresentaram até quatro gêneros de nematóides (Tabela 1).

Tabela 1. Nematóides encontrados associados a espécies de plantas ornamentais e floríferas

\begin{tabular}{|c|c|c|}
\hline Nome científico & Nome comum & Nematóides associados \\
\hline Aegopodium podograria 'Variegatum' & Aegopodium & Meloidogyne sp., Trichodorus sp., Ogma sp. \\
\hline Althernanthera amoena & Periquito & Ogma sp. \\
\hline Anthurium digitatum & Antúrio & Meloidogyne javanica, Xiphinema sp. \\
\hline Asystasia gangetica & Asistásia & Xiphinema sp. \\
\hline Belancanda chinensis & Flor-de-leopardo & $\begin{array}{l}\text { Helicotylenchus dihystera, Meloidogyne sp., Ogma sp., } \\
\text { Xiphinema sp. }\end{array}$ \\
\hline Centaurea gymnocarpa & Centáurea & Helicotylenchus dihystera, Meloidogyne incognita, Ogma sp. \\
\hline Chrysanthemum leucatemum & Margarida & Ditylenchus sp., Meloidogyne incognita \\
\hline Chrysanthemum sp. & Crisântemo & Aphelenchus sp., Dorilaimus sp., Meloidogyne javanica \\
\hline Crassula multicava & Crássula & Meloidogyne incognita \\
\hline Crysanthemum maximum & Margarida & $\begin{array}{l}\text { Helicotylenchus dihystera, Meloidogyne javanica, Ogma sp., } \\
\text { Xiphinema sp. }\end{array}$ \\
\hline Erythrina speciosa & Candelabro & Xiphinema sp., Criconemella sp., Helicotylenchus sp. \\
\hline Graptopetalum paraguayensi & Planta-fantasma & Meloidogyne incognita \\
\hline Hedera helix 'Star' & Hera-verdadeira & Meloidogyne incognita \\
\hline Helicrysum petiolatum & Imortele & $\begin{array}{l}\text { Aphelenchus avenae, Criconemella sp., Meloidogyne } \\
\text { incognita, Trichodorus sp. }\end{array}$ \\
\hline Huernia macrocarpa 'penzigii' & Huérnia & Helicotylenchus dihystera \\
\hline Hypoestes sanguinolenta & Hipoestes & $\begin{array}{l}\text { Meloidogyne sp., Odontonema strictum (Odontonema), } \\
\text { Trichodorus sp., Tylenchorrynchus sp., Xiphinema sp. }\end{array}$ \\
\hline Impatiens walleriana 'Variegata' & Beijinho & Meloidogyne incognita, Helicotylenchus dihystera \\
\hline Iresine lindenili & Iresine & Helicotylenchus dihystera, Meloidogyne javanica \\
\hline Livistona chinensis & Falsa latânia & Helicotylenchus dihystera, Xiphinema sp. \\
\hline Oxalis adenopsis & Trevo-de-quatro-folhas & Meloidogyne incognita \\
\hline Peperomia scandens 'Variegata' & Peperômia & Helicotylenchus dihystera, Meloidogyne sp. \\
\hline Pilea microphylla & Brilhantina & Helicotylenchus dihystera, Meloidogyne incognita \\
\hline Portulaca grandiflora & Onze-horas & $\begin{array}{l}\text { Helicotylenchus sp., Xiphinema, Tylenchorhynchus, } \\
\text { Meloidogyne incognita }\end{array}$ \\
\hline Sedum rubrotinctum & Dedinho-de-moça & Helicotylenchus sp., Meloidogyne javanica \\
\hline Setcreasia purpurea & Trapoeraba-roxa & Meloidogyne sp. \\
\hline Strelitzia reginae & Ave-do-paraíso & $\begin{array}{l}\text { Aphelenchus sp., Trichodorus sp., Tylenchorhynchus sp., } \\
\text { Tylenchulus semipenetrans }\end{array}$ \\
\hline Wedelia trilobata & Margaridinha & Helicotylenchus dihystera, Meloidogyne sp., Trichodorus sp. \\
\hline Zebrina pendula & Tradescância & Criconemella sp., Meloidogyne incognita, Xiphinema sp. \\
\hline
\end{tabular}




\section{REFERÊNCIAS BIBLIOGRÁFICAS}

CAMPOS, V. P. Implicação da sobrevivência dos nematóides em solo e raízes de plantas no controle dos fitopatógenos. Informe Agropecuário, EPAMIG, Belo Horizonte, v.16, n.172, p.15-16, 1992.

CHASE, A. R., KAPLAN, \& OSBORNE, L. S. Nematode pests of tropical foliage plants and leatherleaf. Agriculture Research Education Centre, Apopka, p.83-85, 1983.

COOLEN, D. \& D'HERDE, C. J. A method for the quantitative extration of nematodes from plant tissue. Min. Agric. Res. Adm. State Centre, Ghent, Belgium, 1972. 72p.

CORBETTI, D.D. Three new species of Pratylenchus with a redescription of $P$. andinus. Lordello, Zamith \& Book, 1961 (nematoda: Pratylenchidae). Nematológica, v.29, p.390-403, 1983.

FLEGG, J.J.M. \& HOOPER, D.J. Extration of free-living stages from soil. In: SOUTHEY, J. F. (Ed.) Laboratory methods for work with plant and soil nematodes. London, Ministry of Agriculture: Fisheries and Food . p.5-22, 1970. (Technical Bulletin)

HARTMAN, K.M. \& SASSER, J.N. Identification of Meloidogyne species on the basis of differential host test and perineal-pattern morphology. In: BARKER, K.R.; CARTER, C.C. \& SASSER, J.N. (Eds.) An advanced treatise on Meloidogyne. II. Methodology. North Ca- rolina State University (Department of Plant Pathology): Raleigh, 1985. p.69-77.

INSERRA, R.N.; DUNN, R.A. \& STAN, J.L. Regulatory implications of Rotylenchulus reniformis infestations on ornamental nurseries in Florida. Nursery Digest., v.25, p.18-19, 1981.

JENKINS, W.R. A rapid centrifugal-flotation technique for separating nematodes from soil. Plant Disease Reporter, v.48, p.692, 1964.

LEHRMAN, P.S. \& STOKES, D.E. Effect of the root-knot nematode, Meloidogyne incognita on pothos. Proc. Fla. State Hortic. Cos., v.92, p.343-345, 1979.

LORDELLO, L.G.E. Contribuição ao conhecimento dos nematóides que causam galhas em raízes de plantas do Estado de São Paulo e Estados vizinhos. Anais da Escola Superior de Agricultura Luiz de Queiroz, Piracicaba, v.21, p.182-8. 1964.

MARTIN, K.J. Ocurrence of Radopholus similis and other plant parasitic nematodes in ornamental plants being transported into Arizona. Plant Disease Reporter, v.62, p.293-294, 1978.

SOUTHEY, J.F. Observations on Heterodera cactii Filijev et. sch. Stekhoven and Meloidogyne spp. on imported Cactus plants with a list of new host records. Nematológica, v.11, p.1-6, 1957.

TIHOHOD, D. Nematologia Agrícola Aplicada. Jaboticabal: FUNEP, 1973. $372 \mathrm{p}$. 